University of Nebraska - Lincoln

DigitalCommons@University of Nebraska - Lincoln

$9-2008$

\title{
Incidence of Cancers of the Oral Cavity and Pharynx Among American Indians and Alaska Natives, 1999-2004
}

\author{
Marsha E. Reichman \\ National Cancer Institute, reichmam@mail.nih.gov \\ Janet J. Kelly \\ Alaska Native Tribal Health Consortium, jjkelly@anthc.org \\ Carol L. Kosary \\ National Cancer Institute, kosaryc@mail.nih.gov \\ Steven S. Coughlin \\ Centers for Disease Control and Prevention, Atlanta, sic9@cdc.gov \\ Melissa Jim \\ Centers for Disease Control and Prevention, melissa.jim@ihs.gov \\ See next page for additional authors
}

Follow this and additional works at: https://digitalcommons.unl.edu/publichealthresources

Part of the Public Health Commons

Reichman, Marsha E.; Kelly, Janet J.; Kosary, Carol L.; Coughlin, Steven S.; Jim, Melissa; and Lanier, Anne P., "Incidence of Cancers of the Oral Cavity and Pharynx Among American Indians and Alaska Natives, 1999-2004" (2008). Public Health Resources. 267.

https://digitalcommons.unl.edu/publichealthresources/267

This Article is brought to you for free and open access by the Public Health Resources at DigitalCommons@University of Nebraska - Lincoln. It has been accepted for inclusion in Public Health Resources by an authorized administrator of DigitalCommons@University of Nebraska - Lincoln. 
Authors

Marsha E. Reichman, Janet J. Kelly, Carol L. Kosary, Steven S. Coughlin, Melissa Jim, and Anne P. Lanier 


\section{An Update on Cancer in American Indians and Alaska Natives, 1999-2004}

Supplement to Cancer

\section{Incidence of Cancers of the Oral Cavity and Pharynx Among American Indians and Alaska Natives, 1999-2004}

\author{
Marsha E. Reichman, $\mathrm{PhD}^{1}$ \\ Janet J. Kelly, MS, MPH${ }^{2}$ \\ Carol L. Kosary, MA ${ }^{1}$ \\ Steven S. Coughlin, $\mathrm{PhD}^{3}$ \\ Melissa A. Jim, $\mathrm{MPH}^{3,4}$ \\ Anne P. Lanier, MD, $\mathrm{MPH}^{2}$ \\ ${ }^{1}$ Division of Cancer Control and Population Sciences, \\ National Cancer Institute, Bethesda, Maryland. \\ ${ }^{2}$ Alaska Native Epidemiology Center, Alaska \\ Native Tribal Health Consortium, Anchorage, \\ Alaska. \\ ${ }^{3}$ Division of Cancer Prevention and Control, \\ National Center for Chronic Disease Prevention \\ and Health Promotion, Centers for Disease Con- \\ trol and Prevention, Atlanta, Georgia. \\ ${ }^{4}$ Division of Epidemiology and Disease Prevention, \\ Indian Health Service, Albuquerque, New Mexico.
}

This supplement was sponsored by Cooperative Agreement Number U50 DP424071-04 from the Centers for Disease Control and Prevention, Division of Cancer Prevention and Control.

The findings and conclusions in this report are those of the authors and do not necessarily represent the official position of the Centers for Disease Control and Prevention.

Address for reprints: Marsha E. Reichman, PhD, Surveillance Research Program, Division of Cancer Control and Population Sciences, National Cancer Institute, 6116 Executive Boulevard, Suite 504, Bethesda, MD 20892; Fax: (301) 480-4077; E-mail: ReichmaM@mail.nih.gov

${ }^{*}$ This article is a US Government work and, as such, is in the public domain in the United States of America.

Received May 5, 2008; accepted June 5, 2008.
BACKGROUND. Previous studies identified disparities in incidence rates of cancers of the oral cavity and pharynx between American Indians/Alaska Natives (AI/AN) and non-Hispanic whites (NHW) and differences between various AI/AN populations. Reporting among AI/AN has been hampered by: 1) heterogeneity among various anatomic sites of oral cavity and pharyngeal cancers obscuring unique patterns of individual anatomic sites; 2) race misclassification and underreporting of AI/AN; and 3) sparseness of data needed to identify regional variations.

METHODS. To improve race classification of AI/AN, data from US central cancer registries were linked with Indian Health Service (IHS) records. AI/AN incidence data from 1999 to 2004 were stratified by sex, age, stage at diagnosis, and anatomic subsite for 6 IHS geographic regions and compared with NHW populations.

RESULTS. For all oral cavity and pharynx cancers combined, among residents of Contract Health Service Delivery Area counties, AI/AN overall had significantly lower incidence rates than NHW (8.5 vs 11.0). However, AI/AN rates were significantly higher in the Northern Plains (13.9 vs 10.5) and Alaska (16.3 vs 10.6), significantly lower in the Pacific Coast (7.7 vs 11.6) and Southwest (3.3 vs 10.4), and similar in the Southern Plains (11.4). Overall AI/AN males had higher incidence rates than $\mathrm{AI} / \mathrm{AN}$ women. Nasopharyngeal cancer was more frequent (1.1 AI/AN vs $0.4 \mathrm{NHW}$ ), and tongue cancer less frequent (1.6 AI/AN vs $2.9 \mathrm{NHW}$ ) in $\mathrm{AI} / \mathrm{AN}$ than NHW populations; however, rates varied by region. Stage distribution was modestly less favorable for AI/AN compared with NHW populations.

CONCLUSIONS. Variation by region, anatomic site, and sex indicates a need for research into etiologic factors and attention to regional risk factor profiles when planning cancer control programs. Cancer 2008;113(5 suppl):1256-65. Published 2008 by the American Cancer Society.*

KEYWORDS: cancer, incidence, oral cavity, pharynx, American Indian/Alaska Native, NPCR, SEER.

ancers of the oral cavity and pharynx include malignancies of $\checkmark$ the lip, tongue, floor of mouth, gum and other mouth, salivary gland, oropharynx, tonsil, nasopharynx, hypopharynx, and "other oral cavity and pharynx." Previous studies have identified important disparities in cancers of the oral cavity and pharynx among American Indian/Alaskan Native (AI/AN) populations, and between AI/AN and other populations, including non-Hispanic whites $(\mathrm{NHW}) .^{1-3}$ 
Higher incidence rates have been found for some AI/ AN than NHW populations in the same geographic areas and for males as compared with females. However, previous studies have been hampered by lack of data in individual anatomic cancer sites, misclassification of AI/AN individuals, and small numbers of cases.

The purpose of this study is to improve the characterization of incidence of cancers of the oral cavity and pharynx in the AI/AN population, relative to the NHW population, by using techniques to minimize the effect of race misclassification in cancer surveillance data. This study further examines incidence rates of individual anatomic cancer sites in the oral cancer and pharynx group and stratifies findings by Indian Health Service (IHS) region.

\section{MATERIALS AND METHODS}

Detailed descriptions of the data sources and methods used for this analysis are found in another paper in this supplement. ${ }^{4}$

\section{Cancer Cases}

Population-based central cancer registries collect information on all new cancer cases within their geographic boundaries. They participate in the Surveillance, Epidemiology, and End Results (SEER) program of the National Cancer Institute (NCI), the National Program for Cancer Registries (NPCR) of the Centers for Disease Control and Prevention (CDC), or both. ${ }^{1,5}$ Primary cancer site and histology data are coded according to the International Classification of Diseases for Oncology (ICD-O) edition in use at the time of diagnosis and converted to the Third Edition. ${ }^{6}$ Ten anatomic sites of cancers of the oral cavity and pharynx were defined with the following ICD-O codes: lip (C00.0-C00.9), tongue (C01.9C02.9), gum and other mouth (C03.0-C03.9, C05.0C06.9), floor of the mouth (C04.0-C04.9), salivary gland (C07.9-C08.9), oropharynx (C10.0-C10.9), tonsil (C09.0-C09.9), nasopharynx (C11.0-C11.9), hypopharynx (C12.9-C13.9), and other (C14.0, C14.2-C14.8). Kaposi sarcomas at these sites were excluded, as were histology codes 9590 to 9989, 9050 to 9055, and 9140. All cases of oral cavity and pharynx cancers included in this study were ascertained by population-based cancer registries that agreed to participate and met the United States Cancer Statistics standards for high-quality data within states ${ }^{5}$; the states that met quality criteria are listed in the footnotes of Table 1.

Coding race for AI/AN cancer cases combines information from data linkages with the IHS patient registration database and the multiple race fields in central cancer registry records. ${ }^{7}$ The IHS provides medical services to AI/AN persons who are members of federally recognized tribes. To reduce the misclassification of AI/AN race, central cancer registries worked with the IHS to link all case records with the IHS patient registration database to identify AI/AN cases misclassified as non-Native. Linkages were conducted using LinkPlus, a probabilistic linkage software program developed by CDC. ${ }^{8}$ The information from the linkages was then combined with the multiple race fields coded in cancer registry records. Further details about linkages and coding rules for multiple races are described elsewhere in this Supplement. ${ }^{4}$ To further improve race classification, analyses focused on Contract Health Service Delivery Area (CHSDA) counties (Fig. 1), which generally contain federally recognized tribal lands or are adjacent to tribal lands. The proportions of AI/AN in relation to total population are higher in CHSDA counties than in non-CHSDA counties, and previous analyses indicate less race misclassification for AI/AN in these counties. ${ }^{9}$ About $56 \%$ of the US AI/AN population reside in CHSDA counties. This proportion varies by IHS region: Alaska $=100 \%$; East $=13.1 \%$; Northern Plains $=59.0 \%$; Southern Plains $=64.1 \%$; Pacific Coast $=55.6 \%$; Southwest $=87.5 \%$. In addition, incidence rates were stratified by IHS regions (Alaska, Pacific Coast, Northern Plains, Southern Plains, Southwest, and East) to evaluate the geographic variation of cancer incidence in the AI/AN population (Fig. 1). Additional details about CHSDA counties and IHS are provided elsewhere. ${ }^{4}$

Cancer stage data for this report spanned changes in SEER summary stage coding. Stage was coded according to SEER Summary Stage 1977 rules for diagnosis years 1999 to 2000 and to SEER Summary Stage 2000 rules for 2001 to 2003. The numbers of cases and percent of cases at the local, regional, and distant stages were examined for all instances of differences between SEER Summary Stage 1977 and 2000 for oral cavity and pharynx cancers. Because changes were observed to be minimal, stage data were combined for 1999 to $2003 .{ }^{10,11}$ Collaborative staging was introduced by both NPCR and SEER Programs in 2004. Although SEER summary stage is available through collaborative staging, evaluation of this data for consistency with previously coded data has not been completed, so stage at diagnosis data from 2004 were not included in these analyses.

\section{Population Estimates}

In the 2000 census, the US Census Bureau for the first time allowed respondents to identify themselves 


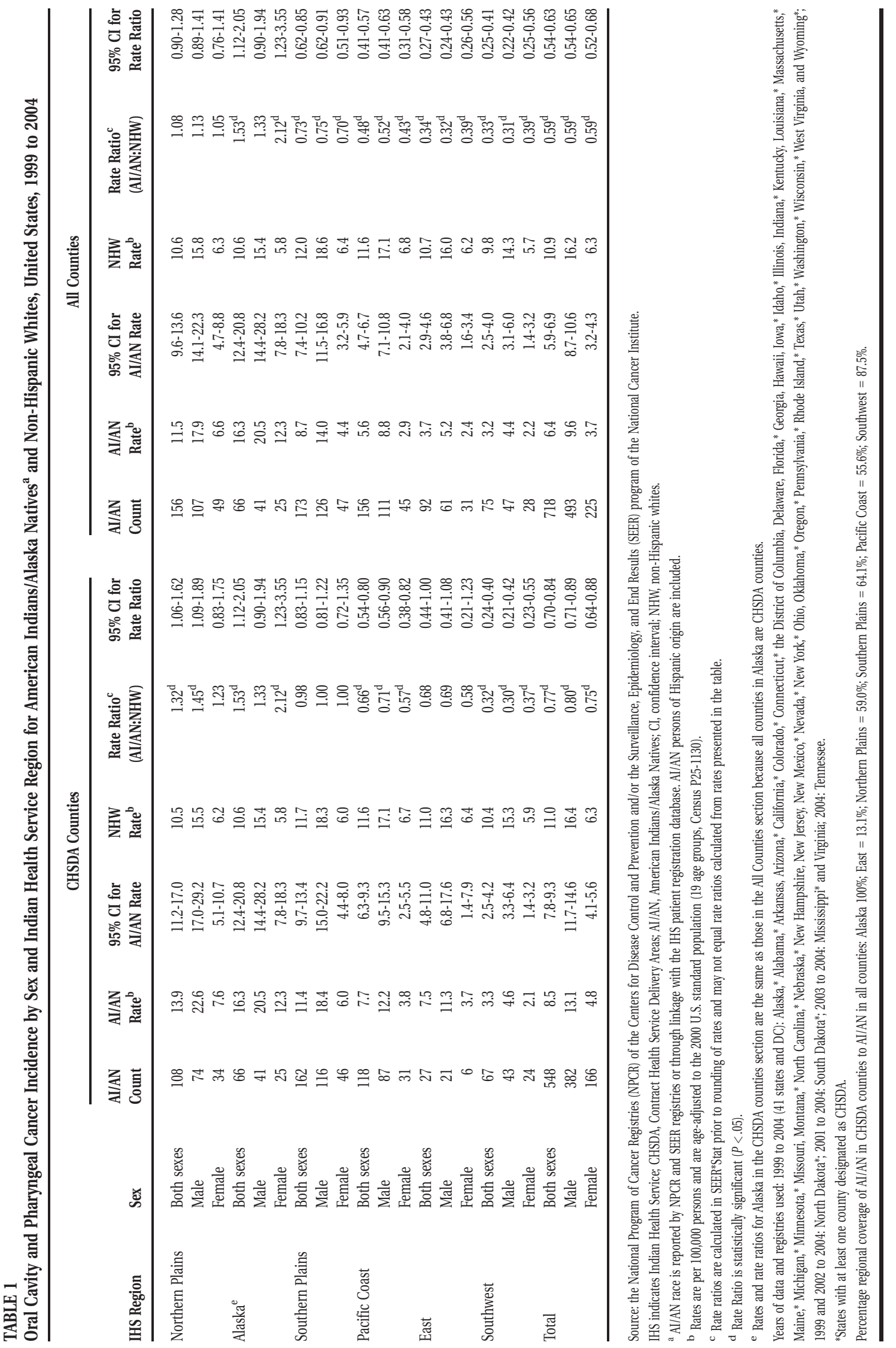




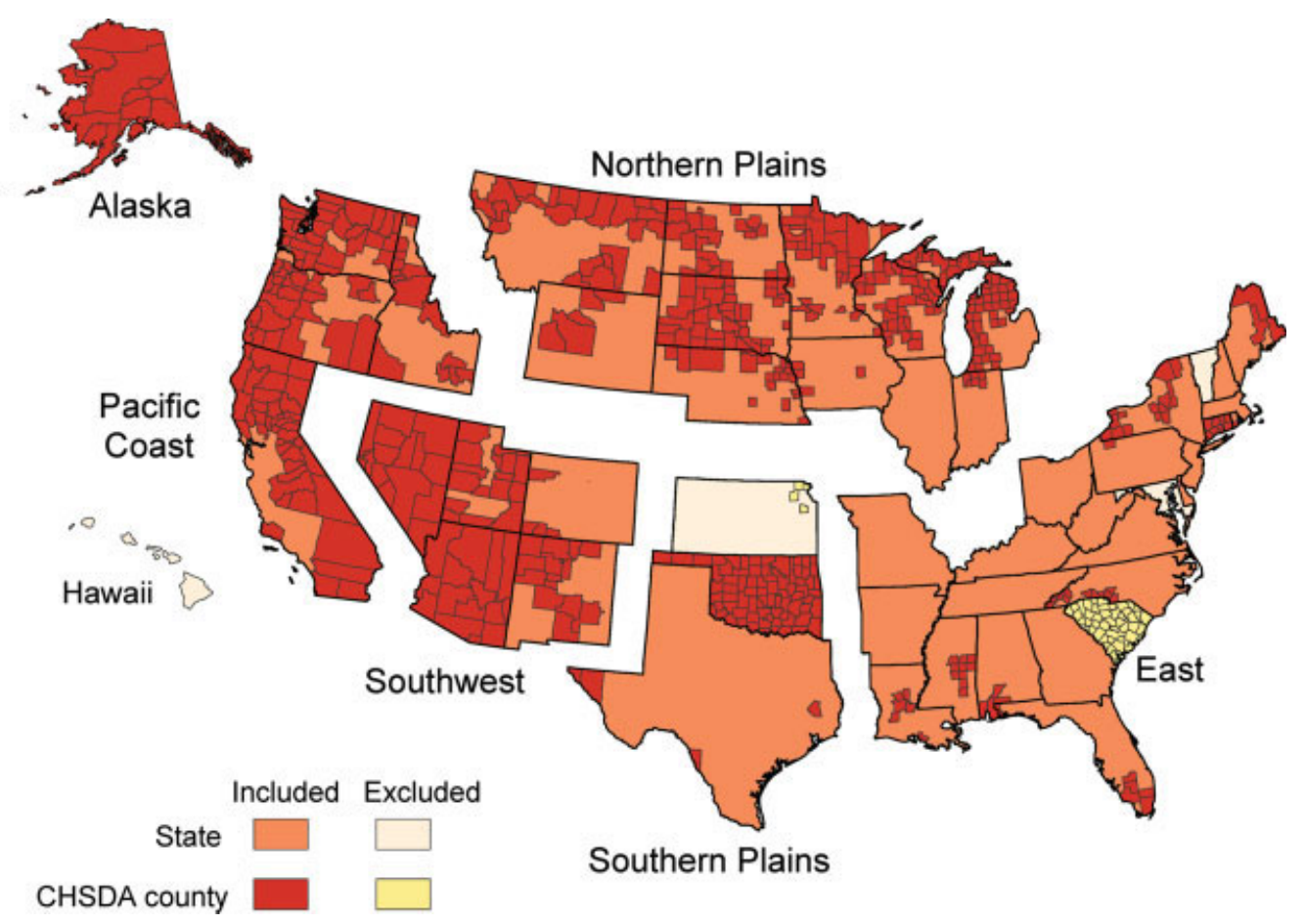

FIGURE 1. States and Contract Health Service Delivery Areas (CHSDA) counties by Indian Health Service Region are depicted.

in 1 or more race fields. ${ }^{7}$ To facilitate comparison with population estimates based on a single race field before 2000, the CDC's National Center for Health Statistics, in collaboration with the Census Bureau, developed a technique of bridging race categories into single-race annual population estimates. ${ }^{12}$ The NCI makes further refinements to these estimates regarding race and county geographic codes; these estimates were used as denominators in this report. $^{13}$

\section{Statistical Analyses}

Cancer incidence rates are expressed per 100,000 persons and are age-adjusted by 19 age groups to the 2000 US standard population. Rates and 95\% modified gamma confidence intervals (CI) are generated using SEER*Stat Software. ${ }^{14,15}$ Percent distributions (stage of disease at diagnosis and data on individual anatomic sites) are also age-adjusted. For presentation, counts are suppressed when the category contains 5 or fewer cases. Groupings for age-specific analyses were selected based on the distribution for all oral cavity and pharyngeal cancer cases combined. Rate ratios (RRs) - the age-adjusted incidence rate among AI/AN divided by the age-adjusted incidence rate among NHW-and 95\% CI were calculated to facilitate regional comparisons of incidence rates between $\mathrm{AI} / \mathrm{AN}$ and NHW populations.

\section{RESULTS}

AI/AN incidence rates for all cancers of the oral cavity and pharynx combined showed substantial regional variation compared with NHW (Table 1). Furthermore incidence rates for all US counties in the dataset were generally lower than those in CHSDA counties where race misclassification is lower. Therefore, subsequent results and discussion are limited to CHSDA counties unless otherwise noted. Over all regions, NHW had a higher incidence rate for cancers of the oral cavity and pharynx (11.0) than did AI/AN (8.5). Among AI/AN, incidence rates for both sexes and all oral cavity and pharynx cancers combined ranged from 3.3 in the Southwest to 16.3 in Alaska. Rates did not vary substantially in the IHS regions among the NHW population. Incidence rates were generally higher for males than females among both AI/AN (male:female rate ratio [RR] 2.7) and NHW (male:female RR, 2.6) for all areas combined. However, there is much more geographic variation in the male:female RR for for AI/AN (from 1.7 in Alaska to 3.2 in the Pacific Coast) than for NHW (range 2.5 to 3.1 ).

The incidence RRs for AI/AN to NHW varied across IHS regions, with AI/AN having significantly higher incidence rates in the Northern Plains and Alaska for both sexes combined, for males in the Northern Plains, and for females in Alaska. In the 
TABLE 2

Oral Cavity and Pharyngeal Cancer Incidence Rates and Percentage Distribution by Anatomic Site and Indian Health Service Region for American Indians/Alaska Natives ${ }^{\mathrm{a}}$ and Non-Hispanic Whites, CHSDA Counties, United States, 1999 to 2004

\begin{tabular}{|c|c|c|c|c|c|c|c|c|c|c|}
\hline \multirow[b]{2}{*}{ Region } & \multirow[b]{2}{*}{ Anatomic Site } & \multicolumn{4}{|c|}{ AI/AN } & \multicolumn{4}{|c|}{ NHW } & \multirow{2}{*}{$\frac{\text { AI/AN:NHW }}{\text { Rate Ratio }}$} \\
\hline & & Count & Rate $^{a}$ & $95 \% \mathrm{CI}$ & $\%$ of Cases ${ }^{b}$ & Count & Rate $^{\mathrm{a}}$ & $95 \% \mathrm{CI}$ & $\%$ of Cases ${ }^{b}$ & \\
\hline \multirow[t]{11}{*}{ Northern Plains } & Lip & 7 & 0.9 & $0.3-1.9$ & 6.5 & 687 & 1.3 & $1.2-1.4$ & 12.4 & 0.68 \\
\hline & Tongue & 19 & 2.5 & $1.5-4.0$ & 18.0 & 1,294 & 2.6 & $2.4-2.7$ & 24.8 & 0.98 \\
\hline & Gum and other mouth & 14 & 2.1 & $1.1-3.7$ & 15.1 & 784 & 1.5 & $1.4-1.6$ & 14.3 & 1.40 \\
\hline & Floor of mouth & 11 & 1.2 & $0.6-2.2$ & 8.6 & 394 & 0.8 & $0.7-0.9$ & 7.6 & 1.55 \\
\hline & Salivary gland & 11 & 1.4 & $0.6-2.6$ & 10.1 & 619 & 1.2 & $1.1-1.3$ & 11.4 & 1.13 \\
\hline & Oropharynx & $\sim$ & 0.5 & $0.1-1.2$ & 3.6 & 205 & 0.4 & $0.4-0.5$ & 3.8 & 1.14 \\
\hline & Tonsil & 21 & 2.3 & $1.4-3.6$ & 16.5 & 663 & 1.3 & $1.2-1.4$ & 12.4 & $1.74^{\mathrm{d}}$ \\
\hline & Nasopharynx & 6 & 0.7 & $0.2-1.5$ & 5.0 & 173 & 0.3 & $0.3-0.4$ & 2.9 & 1.95 \\
\hline & Hypopharynx & 10 & 1.5 & $0.7-2.8$ & 10.8 & 378 & 0.7 & $0.7-0.8$ & 6.7 & 2.04 \\
\hline & Other oral cavity \& pharynx & $\sim$ & 0.8 & $0.2-1.8$ & 5.8 & 127 & 0.2 & $0.2-0.3$ & 1.9 & 3.15 \\
\hline & All oral cavity \& pharynx cancers & 108 & 13.9 & $11.2-17.0$ & 100.0 & 5,324 & 10.5 & $10.2-10.8$ & 100.0 & $1.32^{\mathrm{d}}$ \\
\hline \multirow[t]{11}{*}{ Alaska $^{\mathrm{c}}$} & Lip & $\sim$ & 1.2 & $0.3-3.0$ & 7.4 & 14 & 0.9 & $0.5-1.6$ & 8.5 & 1.32 \\
\hline & Tongue & 12 & 2.8 & $1.4-5.0$ & 17.2 & 64 & 2.9 & $2.2-3.8$ & 27.4 & 0.97 \\
\hline & Gum and other mouth & $\sim$ & 1.3 & $0.4-3.1$ & 8.0 & 22 & 1.2 & $0.7-1.8$ & 11.3 & 1.14 \\
\hline & Floor of mouth & 7 & 1.8 & $0.7-3.7$ & 11.0 & 12 & 0.6 & $0.3-1.0$ & 5.7 & $3.12^{\mathrm{d}}$ \\
\hline & Salivary gland & $\sim$ & 1.1 & $0.3-2.5$ & 6.7 & 22 & 1.1 & $0.7-1.8$ & 10.4 & 0.95 \\
\hline & Oropharynx & $\sim$ & 0.2 & $0.0-1.2$ & 1.2 & 12 & 0.6 & $0.3-1.1$ & 5.7 & 0.35 \\
\hline & Tonsil & $\sim$ & 0.6 & $0.1-1.8$ & 3.7 & 37 & 1.5 & $1.0-2.1$ & 14.2 & 0.38 \\
\hline & Nasopharynx & 27 & 6.8 & $4.4-10.0$ & 41.7 & 17 & 0.8 & $0.4-1.3$ & 7.5 & $8.82^{\mathrm{d}}$ \\
\hline & Hypopharynx & $\sim$ & 0.4 & $0.1-1.6$ & 2.5 & 15 & 0.8 & $0.4-1.3$ & 7.5 & 0.55 \\
\hline & Other oral cavity \& pharynx & $\sim$ & $\sim$ & NA & NA & 6 & 0.3 & $0.1-0.6$ & NA & \\
\hline & All oral cavity \& pharynx cancers & 66 & 16.3 & $12.4-20.8$ & 100.0 & 221 & 10.6 & $9.1-12.3$ & 100.0 & $1.53^{\mathrm{d}}$ \\
\hline \multirow[t]{11}{*}{ Southern Plains } & Lip & 19 & 1.5 & $0.9-2.4$ & 13.2 & 394 & 2.0 & $1.8-2.2$ & 17.1 & 0.77 \\
\hline & Tongue & 26 & 1.9 & $1.2-2.8$ & 16.7 & 512 & 2.6 & $2.4-2.9$ & 22.2 & 0.72 \\
\hline & Gum and other mouth & 24 & 1.8 & $1.1-2.6$ & 15.8 & 313 & 1.6 & $1.4-1.8$ & 13.7 & 1.13 \\
\hline & Floor of mouth & 9 & 0.6 & $0.3-1.2$ & 5.3 & 124 & 0.6 & $0.5-0.7$ & 5.1 & 1.00 \\
\hline & Salivary gland & 12 & 0.8 & $0.4-1.4$ & 7.0 & 260 & 1.3 & $1.2-1.5$ & 11.1 & 0.59 \\
\hline & Oropharynx & $\sim$ & 0.2 & $0.1-0.6$ & 1.8 & 86 & 0.4 & $0.3-0.5$ & 3.4 & 0.57 \\
\hline & Tonsil & 32 & 2.0 & $1.4-2.9$ & 17.5 & 320 & 1.7 & $1.5-1.9$ & 14.5 & 1.21 \\
\hline & Nasopharynx & 15 & 0.9 & $0.5-1.6$ & 7.9 & 86 & 0.5 & $0.4-0.6$ & 4.3 & $2.03^{\mathrm{d}}$ \\
\hline & Hypopharynx & 14 & 1.1 & $0.6-1.8$ & 9.6 & 128 & 0.6 & $0.5-0.8$ & 5.1 & 1.71 \\
\hline & Other oral cavity \& pharynx & 7 & 0.5 & $0.2-1.1$ & 4.4 & 69 & 0.3 & $0.3-0.4$ & 2.6 & 1.55 \\
\hline & All oral cavity \& pharynx cancers & 162 & 11.4 & $9.7-13.4$ & 100.0 & 2,292 & 11.7 & $11.2-12.2$ & 100.0 & 0.98 \\
\hline \multirow{11}{*}{ Pacific Coast } & Lip & 8 & 0.7 & $0.3-1.3$ & 9.1 & 1,183 & 1.3 & $1.2-1.3$ & 11.2 & 0.52 \\
\hline & Tongue & 31 & 2.0 & $1.3-3.0$ & 26.0 & 2,951 & 3.2 & $3.0-3.3$ & 27.6 & $0.65^{\mathrm{d}}$ \\
\hline & Gum and other mouth & 14 & 0.9 & $0.5-1.5$ & 11.7 & 1,544 & 1.6 & $1.6-1.7$ & 13.8 & $0.55^{\mathrm{d}}$ \\
\hline & Floor of mouth & $\sim$ & 0.3 & $0.1-0.7$ & 3.9 & 732 & 0.8 & $0.7-0.8$ & 6.9 & 0.33 \\
\hline & Salivary gland & 8 & 0.5 & $0.2-0.9$ & 6.5 & 1,110 & 1.2 & $1.1-1.3$ & 10.3 & $0.38^{\mathrm{d}}$ \\
\hline & Oropharynx & $\sim$ & 0.1 & $0.0-0.4$ & 1.3 & 319 & 0.3 & $0.3-0.4$ & 2.6 & 0.29 \\
\hline & Tonsil & 24 & 1.6 & $1.0-2.4$ & 20.8 & 1,662 & 1.8 & $1.7-1.9$ & 15.5 & 0.89 \\
\hline & Nasopharynx & 14 & 0.8 & $0.4-1.4$ & 10.4 & 353 & 0.4 & $0.3-0.4$ & 3.4 & $2.14^{\mathrm{d}}$ \\
\hline & Hypopharynx & 10 & 0.7 & $0.3-1.4$ & 9.1 & 721 & 0.8 & $0.7-0.8$ & 6.9 & 0.97 \\
\hline & Other oral cavity \& pharynx & $\sim$ & 0.1 & $0.0-0.5$ & 1.3 & 286 & 0.3 & $0.3-0.3$ & 2.6 & 0.48 \\
\hline & All oral cavity \& pharynx cancers & 118 & 7.7 & $6.3-9.3$ & 100.0 & 10,861 & 11.6 & $11.4-11.8$ & 100.0 & $0.66^{\mathrm{d}}$ \\
\hline \multirow[t]{11}{*}{ East } & Lip & $\sim$ & $\sim$ & NA & NA & 438 & 0.7 & $0.6-0.7$ & NA & $\sim$ \\
\hline & Tongue & 6 & 1.6 & $0.6-3.5$ & 21.3 & 1,900 & 3.0 & $2.8-3.1$ & 27.3 & 0.54 \\
\hline & Gum and other mouth & $\sim$ & 1.8 & $0.5-4.0$ & 24.0 & 1,130 & 1.7 & $1.6-1.8$ & 15.5 & 1.03 \\
\hline & Floor of mouth & $\sim$ & $\sim$ & NA & NA & 514 & 0.8 & $0.7-0.9$ & NA & $\sim$ \\
\hline & Salivary gland & $\sim$ & 1.2 & $0.3-2.9$ & 16.0 & 848 & 1.3 & $1.2-1.4$ & 11.8 & 0.89 \\
\hline & Oropharynx & $\sim$ & 0.2 & $0.0-1.3$ & 2.7 & 317 & 0.5 & $0.4-0.5$ & 4.5 & 0.43 \\
\hline & Tonsil & 8 & 1.8 & $0.8-3.7$ & 24.0 & 962 & 1.5 & $1.4-1.6$ & 13.6 & 1.19 \\
\hline & Nasopharynx & $\sim$ & 0.2 & $0.0-1.4$ & 2.7 & 273 & 0.4 & $0.4-0.5$ & 3.6 & 0.57 \\
\hline & Hypopharynx & $\sim$ & 0.2 & $0.0-1.4$ & 2.7 & 520 & 0.8 & $0.7-0.9$ & 7.3 & 0.29 \\
\hline & Other oral cavity \& pharynx & $\sim$ & 0.5 & $0.0-2.1$ & 6.7 & 233 & 0.4 & $0.3-0.4$ & 3.6 & 1.35 \\
\hline & All oral cavity \& pharynx cancers & 27 & 7.5 & $4.8-11.0$ & 100.0 & 7,135 & 11.0 & $10.7-11.2$ & 100.0 & $\begin{array}{r}0.68 \\
\text { (continued) }\end{array}$ \\
\hline
\end{tabular}


TABLE 2

(continued)

\begin{tabular}{|c|c|c|c|c|c|c|c|c|c|c|}
\hline \multirow[b]{2}{*}{ Region } & \multirow[b]{2}{*}{ Anatomic Site } & \multicolumn{4}{|c|}{$\mathrm{AI} / \mathrm{AN}$} & \multicolumn{4}{|c|}{ NHW } & \multirow{2}{*}{$\frac{\text { AI/AN:NHW }}{\text { Rate Ratio }^{\mathrm{d}}}$} \\
\hline & & Count & Rate $^{\mathrm{a}}$ & $95 \% \mathrm{CI}$ & $\%$ of Cases ${ }^{b}$ & Count & Rate $^{\mathrm{a}}$ & $95 \% \mathrm{CI}$ & $\%$ of Cases ${ }^{b}$ & \\
\hline \multirow[t]{11}{*}{ Southwest } & Lip & $\sim$ & 0.2 & $0.1-0.5$ & 6.1 & 413 & 1.0 & $0.9-1.1$ & 9.6 & 0.22 \\
\hline & Tongue & 7 & 0.4 & $0.1-0.7$ & 12.1 & 1,238 & 2.9 & $2.8-3.1$ & 27.9 & $0.12^{\mathrm{d}}$ \\
\hline & Gum and other mouth & 8 & 0.4 & $0.2-0.8$ & 12.1 & 578 & 1.3 & $1.2-1.5$ & 12.5 & $0.32^{\mathrm{d}}$ \\
\hline & Floor of mouth & $\sim$ & 0.2 & $0.0-0.5$ & 6.1 & 340 & 0.8 & $0.7-0.9$ & 7.7 & 0.24 \\
\hline & Salivary gland & 22 & 1.0 & $0.6-1.6$ & 30.3 & 500 & 1.2 & $1.1-1.3$ & 11.5 & 0.88 \\
\hline & Oropharynx & $\sim$ & $\sim$ & NA & NA & 150 & 0.3 & $0.3-0.4$ & NA & $\sim$ \\
\hline & Tonsil & $\sim$ & 0.2 & $0.1-0.5$ & 6.1 & 589 & 1.4 & $1.3-1.5$ & 13.5 & 0.15 \\
\hline & Nasopharynx & 11 & 0.5 & $0.2-0.9$ & 15.2 & 162 & 0.4 & $0.3-0.5$ & 3.8 & 1.19 \\
\hline & Hypopharynx & $\sim$ & 0.3 & $0.1-0.7$ & 9.1 & 292 & 0.7 & $0.6-0.7$ & 6.7 & 0.43 \\
\hline & Other oral cavity \& pharynx & $\sim$ & 0.1 & $0.0-0.3$ & 3.0 & 150 & 0.3 & $0.3-0.4$ & 2.9 & 0.19 \\
\hline & All oral cavity \& pharynx cancers & 67 & 3.3 & $2.5-4.2$ & 100.0 & 4,412 & 10.4 & $10.1-10.7$ & 100.0 & $0.32^{\mathrm{d}}$ \\
\hline \multirow[t]{11}{*}{ Total } & Lip & 43 & 0.8 & $0.5-1.0$ & 9.4 & 3,129 & 1.1 & $1.1-1.2$ & 10.0 & $0.67^{\mathrm{d}}$ \\
\hline & Tongue & 101 & 1.6 & $1.3-2.0$ & 18.8 & 7,959 & 2.9 & $2.9-3.0$ & 26.4 & $0.55^{\mathrm{d}}$ \\
\hline & Gum and other mouth & 70 & 1.2 & $0.9-1.5$ & 14.1 & 4,371 & 1.6 & $1.5-1.6$ & 14.5 & $0.76^{\mathrm{d}}$ \\
\hline & Floor of mouth & 34 & 0.5 & $0.4-0.8$ & 5.9 & 2,116 & 0.8 & $0.7-0.8$ & 7.3 & $0.70^{\mathrm{d}}$ \\
\hline & Salivary gland & 62 & 0.9 & $0.7-1.2$ & 10.6 & 3,359 & 1.2 & $1.2-1.3$ & 10.9 & $0.73^{\mathrm{d}}$ \\
\hline & Oropharynx & 12 & 0.2 & $0.1-0.3$ & 2.4 & 1,089 & 0.4 & $0.4-0.4$ & 3.6 & $0.41^{\mathrm{d}}$ \\
\hline & Tonsil & 93 & 1.3 & $1.0-1.6$ & 15.3 & 4,233 & 1.6 & $1.5-1.6$ & 14.5 & 0.82 \\
\hline & Nasopharynx & 74 & 1.1 & $0.8-1.4$ & 12.9 & 1,064 & 0.4 & $0.4-0.4$ & 3.6 & $2.68^{\mathrm{d}}$ \\
\hline & Hypopharynx & 42 & 0.7 & $0.5-1.0$ & 8.2 & 2,054 & 0.7 & $0.7-0.8$ & 6.4 & 0.99 \\
\hline & Other oral cavity \& pharynx & 17 & 0.3 & $0.2-0.5$ & 3.5 & 871 & 0.3 & $0.3-0.3$ & 2.7 & 0.96 \\
\hline & All oral cavity \& pharynx cancers & 548 & 8.5 & $7.8-9.3$ & 100.0 & 30,245 & 11.0 & $10.9-11.2$ & 100.0 & $0.77^{\mathrm{d}}$ \\
\hline
\end{tabular}

Source: the National Program of Cancer Registries of the Centers for Disease Control and Prevention and/or the Surveillance, Epidemiology, and End Results program of the National Cancer Institute; see Table 1 for states included.

CHSDA indicates Contract Health Service Delivery Areas; NHW, non-Hispanic whites; AI/AN, American Indians/Alaska Natives; CI, confidence interval; NA, not available.

${ }^{a}$ Rates are per 100,000 persons and are age-adjusted to the 2000 U.S. standard population (19 age groups, Census P25-1130).

b Percentage anatomic site distribution is age-adjusted to the 2000 U.S. standard population and may not add to $100.0 \%$ due to rounding.

${ }^{\mathrm{c}}$ Rates and rate ratios for Alaska in the CHSDA counties section is the same as those in the All Counties section because all counties in Alaska are CHSDA counties.

${ }^{\mathrm{d}}$ Rate ratio is statistically significant $(P<.05)$.

$\sim$ Counts less than 6 are suppressed; if no cases were reported, then row percentages, rates, and rate ratios could not be calculated.

Southern Plains AI/AN and NHW incidence rates are comparable. For other regions and for all regions combined AI/AN incidence rates are lower than NHW rates, reaching significance in each region, for males, females, and both sexes combined except in the East.

Incidence rates, counts, percent distributions, and RRs for individual anatomic sites of oral cavity and pharynx cancers in the 6 IHS regions and for all regions combined for CHSDA counties are shown in Table 2. For all regions combined, AI/AN have a significantly lower incidence rate of cancer of the tongue (1.6) than NHW (2.9), whereas AI/AN have a significantly higher rate of nasopharyngeal cancer (1.1) than NHW (0.4). Rates for other anatomic sites are generally modestly lower in AI/AN populations for all regions combined, reaching significance for cancer of the lip, gum and other mouth, floor of mouth, salivary gland, and oropharynx. When analyzed by region, significantly higher AI/AN incidence rates of nasopharyngeal cancer compared with NHW are noted in Alaska (RR, 8.82), the Southern Plains (RR, 2.03), and the Pacific Coast (RR, 2.14). AI/AN incidence rates are significantly higher than NHW rates for tonsillar cancer in the Northern Plains (RR, 1.74) and for cancer of the floor of the mouth in Alaska (RR, 3.12). The incidence rate for cancer of the tongue is similar among $\mathrm{AI} / \mathrm{AN}$ and NHW in Alaska and the Northern Plains and generally lower for AI/AN than NHW elsewhere, with significance reached in the Southwest and Pacific Coast. Other oral cavity and pharynx cancers are generally diagnosed less frequently among AI/AN than NHW.

The age at diagnosis for oral cavity and pharynx cancers is lower for $\mathrm{AI} / \mathrm{AN}$ than for NHW, with $36.3 \%$ of cases in AI/AN diagnosed at age 65 years or older as compared with $48.8 \%$ of cases among NHW (Table 3 ). This pattern holds true for all regions of the country except for Alaska, where the percentage of cases diagnosed at age 65 years or older is $39.4 \%$ for AI/AN and $35.7 \%$ for NHW. In general, the pattern of younger age at diagnosis among AI/AN than NHW 
TABLE 3

Oral Cavity and Pharyngeal Cancer Incidence Rates and Percentage Distribution by Age and Indian Health Service Region for American Indians/Alaska Natives and Non-Hispanic Whites, CHSDA Counties, United States, 1999 to 2004

\begin{tabular}{|c|c|c|c|c|c|c|c|c|c|c|c|c|c|c|c|c|}
\hline \multirow[b]{2}{*}{ IHS Region } & \multicolumn{4}{|c|}{$<50$ Years } & \multicolumn{4}{|c|}{ 50-64 Years } & \multicolumn{4}{|c|}{$65+$ Years } & \multicolumn{4}{|c|}{ Total } \\
\hline & Count & Row $\%^{\mathrm{a}}$ & Rate $^{b}$ & $95 \% \mathrm{CI}$ & Count & Row \% ${ }^{\mathrm{a}}$ & Rate $^{\mathrm{b}}$ & $95 \% \mathrm{CI}$ & Count & Row $\%^{a}$ & Rate $^{\mathrm{b}}$ & $95 \% \mathrm{CI}$ & Count & Row $\%^{a}$ & Rate $^{\mathrm{b}}$ & $95 \% \mathrm{CI}$ \\
\hline \multicolumn{17}{|c|}{ American Indian/Alaska Native } \\
\hline Northern Plains & 21 & 19.4 & 2.3 & $1.4-3.5$ & 42 & 38.9 & 27.9 & $20.1-37.7$ & 45 & 41.7 & $63.7^{\mathrm{c}}$ & $45.8-86.3$ & 108 & 100 & $13.9^{\mathrm{c}}$ & $11.2-17.0$ \\
\hline Alaska & 12 & 18.2 & 2.8 & $1.5-4.9$ & 28 & 42.4 & $39.4^{\mathrm{c}}$ & $26.1-57.0$ & 26 & 39.4 & 65.7 & $42.4-97.7$ & 66 & 100 & $16.3^{\mathrm{c}}$ & $12.4-20.8$ \\
\hline Southern Plains & 42 & 25.9 & 3.3 & $2.4-4.5$ & 61 & 37.7 & 25.3 & $19.4-32.5$ & 59 & 36.4 & 41.3 & $31.4-53.4$ & 162 & 100 & 11.4 & $9.7-13.4$ \\
\hline Pacific Coast & 33 & 28.0 & 2.1 & $1.5-3.0$ & 50 & 42.4 & $18.4^{\mathrm{d}}$ & $13.7-24.3$ & 35 & 29.7 & $26.8^{\mathrm{d}}$ & $18.4-37.8$ & 118 & 100 & $7.7^{\mathrm{d}}$ & $6.3-9.3$ \\
\hline East & 6 & 22.2 & 1.7 & $0.6-3.7$ & 12 & 44.4 & 19.0 & $9.8-33.1$ & 9 & 33.3 & 26.8 & $12.1-51.8$ & 27 & 100 & 7.5 & $4.8-11.0$ \\
\hline Southwest & 21 & 31.3 & $1.0^{\mathrm{d}}$ & $0.6-1.5$ & 21 & 31.3 & $6.3^{\mathrm{d}}$ & $3.9-9.7$ & 25 & 37.3 & $12.7^{\mathrm{d}}$ & $8.1-19.0$ & 67 & 100 & $3.3^{\mathrm{d}}$ & $2.5-$ \\
\hline Total & 135 & 24.6 & $2.1^{\mathrm{d}}$ & $1.7-2.4$ & 214 & 39.1 & $19.0^{\mathrm{d}}$ & $16.5-21.7$ & 199 & 36.3 & $33.1^{\mathrm{d}}$ & $28.6-38.1$ & 548 & 1000 & $8.5^{\mathrm{d}}$ & $7.8-9.3$ \\
\hline \multicolumn{17}{|c|}{ Non-Hispanic white } \\
\hline Northern Plains & 877 & 16.5 & 2.6 & $2.4-2.8$ & 1,857 & 34.9 & 24.2 & 23.1-25.3 & 2,590 & 48.6 & 39.5 & $38.0-41.0$ & 5,324 & 100 & 10.5 & $10.2-10.8$ \\
\hline Alaska & 53 & 24.0 & 2.3 & $1.7-3.0$ & 89 & 40.3 & 20.5 & $16.4-25.2$ & 79 & 35.7 & 46.9 & $36.9-58.7$ & 221 & 100 & 10.6 & $9.1-12.3$ \\
\hline Southern Plains & 364 & 15.9 & 3.0 & $2.7-3.4$ & 749 & 32.7 & 25.0 & 23.3-26.9 & 1,179 & 51.4 & 45.2 & $42.7-47.9$ & 2,292 & 100 & 11.7 & $11.2-12.2$ \\
\hline Pacific Coast & 1,652 & 15.2 & 2.7 & $2.5-2.8$ & 3,861 & 35.5 & 26.6 & $25.8-27.5$ & 5,348 & 49.2 & 44.9 & $43.7-46.2$ & 10,861 & 100 & 11.6 & $11.4-11.8$ \\
\hline East & 1,101 & 15.4 & 2.8 & $2.6-3.0$ & 2,532 & 35.5 & 26.5 & $25.5-27.5$ & 3,502 & 49.1 & 39.4 & $38.1-40.8$ & 7,135 & 100 & 11.0 & $10.7-11.2$ \\
\hline Southwest & 616 & 14.0 & 2.5 & $2.3-2.7$ & 1,732 & 39.3 & 26.5 & $25.3-27.8$ & 2,064 & 46.8 & 36.3 & $34.8-37.9$ & 4,412 & 100 & 10.4 & $10.1-10.7$ \\
\hline Total & 4,663 & 15.4 & 2.7 & $2.6-2.8$ & 10,820 & 35.8 & 25.9 & $25.4-26.4$ & 14,762 & 48.8 & 41.2 & $40.6-41.9$ & 30,245 & 100 & 11.0 & $10.9-11.2$ \\
\hline
\end{tabular}

Source: the National Program of Cancer Registries of the Centers for Disease Control and Prevention and/or the Surveillance, Epidemiology, and End Results program of the National Cancer Institute; see Table 1 for states included.

CHSDA indicates Contract Health Service Delivery Areas; IHS, Indian Health Service; CI: confidence interval.

${ }^{\text {a }}$ Percentages may not add up to $100.0 \%$ due to rounding.

${ }^{\mathrm{b}}$ Rates are per 100,000 persons and are age-adjusted to the 2000 US standard population (19 age groups, Census P25-1130).

c The American Indian/Alaska Native (AI/AN) rate is statistically significantly higher than the non-Hispanic white (NHW) rate $(P<.05)$.

${ }^{\mathrm{d}}$ AI/AN rate is statistically significantly lower than the NHW rate $(P<.05)$.

$\sim$ Counts less than 6 are suppressed; if no cases were reported, then row percentages and rates could not be calculated.

holds true when cancers at individual anatomic sites (except nasopharyngeal cancer) are examined (data not shown). Exceptions occur for tongue cancer in the Northern Plains, the Southern Plains, and Alaska and for hypopharynx cancer in the Northern Plains.

For those residing in CHSDA counties, stage at diagnosis was examined for all cancers of the oral cavity and pharynx combined (Table 4). Comparing AI/AN stage distribution to that for NHW for all regions combined, the percentage of cancers diagnosed at the local stage is modestly higher for NHW, similar for the regional stage, and modestly lower for NHW at the distant stage. For Alaska, $28.6 \%$ of cancers are diagnosed at the distant stage for AI/AN as compared with $7.5 \%$ for NHW. Similarly, in the Southwest, distant stage diagnoses comprise $18.8 \%$ of cancer cases for AI/AN but only $8.6 \%$ for NHW. Diagnoses at the localized stage are notably lower for AI/AN than for NHW in Alaska (24.6\% vs $35.8 \%)$ and the Northern Plains (30.7\% vs $42.1 \%)$. When stage is examined by individual anatomic sites, similar patterns are observed (data not shown). However, for cancer of the tonsil, a greater percentage of cases among $\mathrm{AI} / \mathrm{AN}$ are diagnosed at the local stage
(24.7\%) than among NHW (15.5\%). Salivary gland cancer is similarly diagnosed more frequently at earlier stages in $\mathrm{AI} / \mathrm{AN}(64.7 \%)$ than in NHW (44.6\%).

\section{DISCUSSION}

The incidence rate for all cancers of the oral cavity and pharynx combined among AI/AN in CHSDA counties in the US is lower than among NHW (8.5 vs 11.0). However, detailed analyses by individual anatomic cancer sites and/or by IHS geographic regions show a much more varied picture. In particular, cancers of the tongue make up a smaller, whereas nasopharyngeal cancers make up a much larger, proportion of these cancers in AI/AN than NHW. In Alaska, the incidence rates for all cancers of the oral cavity and pharynx is significantly higher for AI/AN than for NHW (RR, 1.53). In addition, nasopharyngeal cancer stands out as having significantly higher incidence rates among AI/AN than NHW in Alaska, the Southern Plains, and the Pacific Coast. Nasopharyngeal cancers comprise $42 \%$ of all Alaskan AI/AN cancers of the oral cavity and pharynx. Other instances of significantly higher incidence rates 


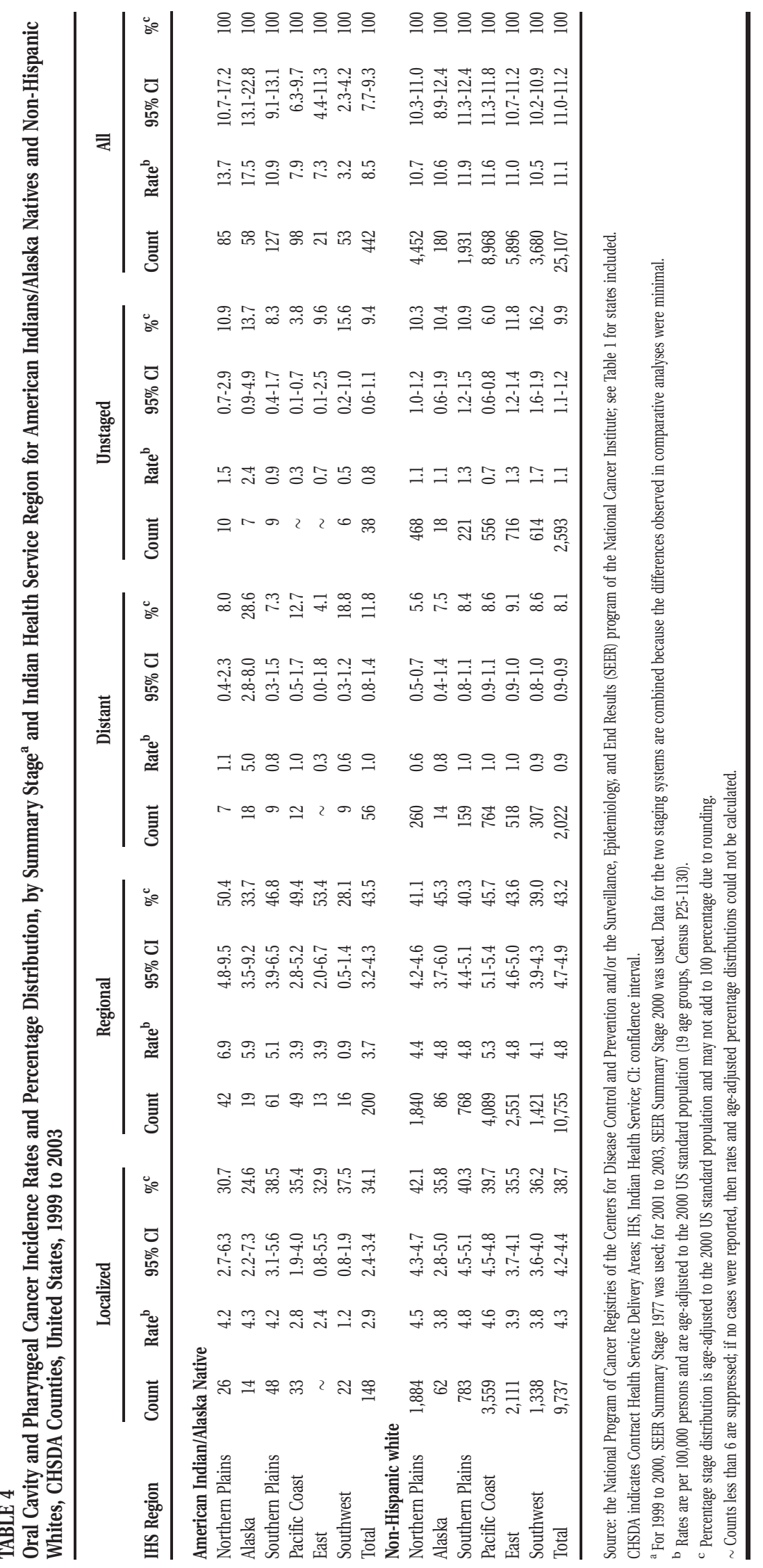


among AI/AN than NHW in the same geographic region are indicated above. It is important to take differences revealed in the detailed analyses into account when describing the burden of cancer and in cancer control planning. The age at diagnosis for all cancers of the oral cavity and pharynx was found to be lower for AI/AN than for NHW.

Tobacco use is by far the greatest risk factor for oral cavity and pharynx cancers. ${ }^{16-18}$ AI/AN are more likely to be current smokers $(31.1 \%)$ than NHW (22.8\%), as reported by the Behavioral Risk Factor Surveillance Surveys (BRFSS) for years 2000 to 2006. ${ }^{19}$ High rates of oral cavity and pharynx cancers among AI/AN correspond with high smoking prevalence estimates with 1 exception. Smoking prevalence in the East $(36.5 \%)$ is high; however, the overall rate of oral cavity and pharynx cancer is among the lowest (7.5 per 100,000). However, the East (Fig. 1) has a low percentage of CHSDA counties, which may affect external validity of cancer incidence data. In the Southwest, where the prevalence of current smoking is low $(21.1 \%),{ }^{19}$ cancer rates for oral cavity and pharynx are also low (3.3 per 100,000). Oral cavity and pharynx cancers are up to 5 times more common in drinkers than nondrinkers. ${ }^{17,20}$ Alcohol strongly increases a smoker's risk of cancer of the oral cavity and pharynx, possibly by increasing the exposure to carcinogens. ${ }^{21-23}$ BRFSS 2000 to $2006^{19}$ data indicate a similar pattern across IHS regions of self-reported heavy drinking among AI/AN groups (range, 3\%-7\%) and NHW (6\%).

Other major risk factors for oral cavity and pharynx cancers include a diet low in fruits and vegetables and, for some anatomic sites, viral infection. ${ }^{17,24}$ $\mathrm{AI} / \mathrm{AN}$ and NHW persons reported to BRFSS a similar prevalence of consuming 5 or more servings of fruits and vegetables per day with little variation by IHS region. ${ }^{19}$ Risk factors also vary for individual anatomic sites. Exposures to sunlight, outdoor occupation, and rural residence have been identified as risk factors for lip cancer. Human papilloma virus has been associated with cancers of the tonsil, base of the tongue, and oropharynx. ${ }^{25,26}$ The incidence patterns of oral cavity and pharyngeal cancers observed in the present study may reflect racial/ethnic and regional differences in exposure to these and other factors.

Epstein-Barr virus (EBV) is almost always found in nasopharyngeal cancer cells. ${ }^{27}$ Infection with EBV alone is not sufficient to cause nasopharyngeal cancer. In studies primarily of Asian populations, nasopharyngeal cancer has been found to be associated with consumption of a variety of preserved foods beginning at an early age. ${ }^{28}$ Occupation exposures to formaldehyde, wood dust, and wood preservatives may increase risk for nasopharyngeal cancer. ${ }^{29,30}$ This study shows that Alaska Native people have uniquely increased risk compared with other AI/AN populations in the United States. EBV has been confirmed present in nasopharyngeal cancer tissues among Alaska Natives, ${ }^{31}$ but the role of genetic, environmental, and diet factors in AI/AN persons in Alaska has not been identified.

The presentation of incidence rates for individual anatomic sites for cancers of the oral cavity and pharynx for the 6 IHS regions is possible because of the matching of NPCR and SEER data with the IHS health database, thereby enhancing classification of AI/AN individuals. Limitations of this linkage include exclusion of AI/AN cases from areas with little or no provision of IHS medical services, including many urban areas. Also, some individuals who are AI/AN may access healthcare from other sources. In general, AI/AN are much more accurately classified in CHSDA counties than in non-CHSDA counties. For this reason, CHSDA counties form the basis of most analyses performed here. The availability of more detailed data on oral cavity and pharynx cancers in AI/AN populations will provide a better estimation of the burden of cancer, allow a more targeted allocation of cancer control resources, and facilitate further examination of trends in incidence as well as analysis of survival, morbidity, and mortality.

\section{REFERENCES}

1. Ries L, Melbert D, Krapcho M, et al. SEER cancer statistics review, 1975-2004. Bethesda, MD: National Cancer Institute; 2007. Available at: http://seer.cancer.gov/csr/1975_2004/ Accessed on July 7, 2008.

2. Paltoo DN, Chu KC. Patterns in cancer incidence among American Indians/Alaska Natives, United States, 1992-1999. Public Health Rep. 2004;119:443-451.

3. Espey DK, Wu XC, Swan J, et al. Annual report to the nation on the status of cancer, 1975-2004, featuring cancer in American Indians and Alaska Natives. Cancer. 2007;110: 2119-2152.

4. Espey DK, Wiggins CL, Jim MA, Miller BA, Johnson CJ, Becker TM. Methods for improving cancer surveillance data in American Indian and Alaska Native populations. Cancer. 2008;113(5 suppl):1120-1130.

5. US Cancer Statistics Working Group. United States cancer statistics: 2003 incidence and mortality. Atlanta, GA: US Department of Health and Human Services, Centers for Disease Control and Prevention and National Cancer Institute; 2006.

6. Fritz A, Percy C, Jack A. International classification of diseases of oncology. Geneva, Switzerland: World Health Organization; 2000.

7. Office of Management and Budget. Revisions to the Standards for the Classification of Federal Data on Race and Ethnicity. Federal Register Notice: October 30, 1997. Available at: http://www.whitehouse.gov/omb/fedreg/1997standards. html Accessed on July 7, 2008. 
8. Centers for Disease Control and Prevention. Link Plus: A suite of publicly available software programs for collecting and processing cancer registry data. 2005. Available at: http://www.cdc.gov/cancer/npcr/tools/registryplus/lp.htm Accessed on July 7, 2008.

9. Jim MA, Espey DK, Wiggins CL, Cobb N, Wingo PA. Misclassification of American Indians Residing Near IHS Facilities, Poster P-47, Final Program and Abstracts. The North American Association of Central Cancer Registries Annual Conference; 2006.

10. Howe HL, Jamison M, Havener L, Chen VW, Ries LAG. Sitespecific comparison of summary stage 1977 and summary stage 2000 coding. Available at: http://www.naaccr.org/ index.asp?Col_SectionKey=11\&Col_ContentID=397.

11. Phillips J. Summary stage: data effects of the changes in 2000. Available at: http://www.naaccr.org/filesystem/ pdf/Summary\%20Stage\%20Report\%201-21-04b.pdf Accessed on July 7, 2008.

12. Ingram DD, Parker JD, Schenker $\mathrm{N}$, et al. United States Census 2000 population with bridged race categories. Vital Health Stat 2. September 2003:1-55.

13. National Cancer Institute. Surveillance, Epidemiology, and End Results (SEER) program. Statistical esources. U.S. population data 1969-2004. Available at: http://seer.cancer. gov/resources Accessed on July 7, 2008.

14. National Cancer Institute, DCCPS, Surveillance Research Program, Cancer Statistics Branch. SEER*Stat software. 2007. Available at: http://www.seer.cancer.gov/seerstat Accessed on July 7, 2008.

15. Tiwari RC, Clegg LX, Zou Z. Efficient interval estimation for age-adjusted cancer rates. Stat Methods Med Res. 2006;15: 547-569.

16. National Cancer Institute. Changes in cigarette-related disease risks and their implication for prevention and control. NIH Publication No. 97-4213. Bethesda, MD: National Cancer Institute; 1997.

17. Blot WJ, McLaughlin JK, Winn DM, et al. Smoking and drinking in relation to oral and pharyngeal cancer. Cancer Res. 1988;48:3282-3287.

18. Nam JM, McLaughlin JK, Blot WJ. Cigarette smoking, alcohol, and nasopharyngeal carcinoma: a case-control study among U.S. whites. J Natl Cancer Inst. 1992;84:619-622.

19. Steele C, Cardinez C, Richardson L, Tom-Orme L, Shaw K. Surveillance for health behaviors of American Indians and Alaska Natives-findings from the Behavioral Risk Factor
Surveillance System, 2000-2006. Cancer. 2008;113(5 suppl): 1131-1141.

20. Castellsague X, Munoz N. Re: Cancer of the oral cavity and pharynx in nonsmokers who drink alcohol and in nondrinkers who smoke tobacco. J Natl Cancer Inst. 1999;91:13361338.

21. Zheng TZ, Boyle P, Hu HF, et al. Tobacco smoking, alcohol consumption, and risk of oral cancer: a case-control study in Beijing, People's Republic of China. Cancer Causes Control. 1990;1:173-179.

22. Franceschi S, Levi F, La Vecchia C, et al. Comparison of the effect of smoking and alcohol drinking between oral and pharyngeal cancer. Int J Cancer. 1999;83:1-4.

23. Mashberg A, Boffetta P, Winkelman R, Garfinkel L. Tobacco smoking, alcohol drinking, and cancer of the oral cavity and oropharynx among U.S. veterans. Cancer. 1993;72:1369-1375.

24. American Cancer Society. Oral cavity and oropharyngeal cancer detailed guide. 2007. Available at: http://documents. cancer.org/5043.00/5043.00.pdf 2007 Accessed on July 7, 2008.

25. Herrero R, Castellsague X, Pawlita M, et al. Human papillomavirus and oral cancer: the International Agency for Research on Cancer multicenter study. J Natl Cancer Inst. 2003;95:1772-1783.

26. Ryerson AB, Peters ES, Coughlin SS, et al. Burden of potentially HPV-associated cancers of the oropharynx and oral cavity in the U.S., 1998-2003. Cancer. 2008 (in press).

27. IARC. IARC Monographs on the evaluation of carcinogenic risks to humans. Vol 70. Epstein-Barr virus and Kaposi's sarcoma herpesvirus/human herpesvirus 9. Lyon, France: International Agency for Research on Cancer; 1997.

28. Yu MC, Mo CC, Chong WX, Yeh FS, Henderson BE. Preserved foods and nasopharyngeal carcinoma: a case-control study in Guangxi, China. Cancer Res. 1988;48:1954-1959.

29. Hildesheim A, Dosemeci M, Chan CC, et al. Occupational exposure to wood, formaldehyde, and solvents and risk of nasopharyngeal carcinoma. Cancer Epidemiol Biomarkers Prev. 2001;10:1145-1153.

30. IARC. IARC Monographs on the evaluation of carcinogenic risks to humans. Vol 62. Wood dust, formaldehyde. Lyon, France: International Agency for Research on Cancer; 1995.

31. Lanier AP, Bornkamm GW, Henle W, et al. Association of Epstein-Barr virus with nasopharyngeal carcinoma in Alaskan native patients: serum antibodies and tissue EBNA and DNA. Int J Cancer. 1981;28:301-305. 